\title{
Millifluidic Sensor Dedicated to the Microwave Dielectric Spectroscopy of Liquids
}

\author{
P. Jaque Gonzalez ${ }^{\# 1}$, D. Dubuc ${ }^{\# 2}$, K. Grenier ${ }^{\# 3}$, T. Veronese ${ }^{* 4}$ \\ \#LAAS-CNRS, Toulouse, France \\ *Ovalie-Innovation, Auch, France \\ $\left\{{ }^{1}\right.$ pfjaqueg, ${ }^{2}$ dubuc, ${ }^{3}$ grenier $\} @$ laas.fr, ${ }^{4}$ thierry.veronese@ ovalie-innovation.com
}

\begin{abstract}
This paper presents a microwave sensor, which has been designed to dielectrically characterize liquids in the frequency range of 0.1 to $4 \mathrm{GHz}$ for a fluid volume of the order of $1 \mathrm{ml}$. Accuracy of the technique has been evaluated for two case studies. One is based on BSA molecules in aqueous solution at different concentrations, whereas the second case study is related to the dielectric measurements of eggs constituents. Even if these different liquids may present intrinsic variability due to the living, a weak standard deviation of 0.01 for BSA solutions and 0.05 for extra-embryonic liquids have been reached respectively. This accuracy enables the future use of the sensor and measurement technique in various applications and notably for the agriculture of precision, which mostly requires ubiquitous and affordable analytic equipment.
\end{abstract}

Keywords - microwave spectroscopy, coplanar waveguides, biosensors, millifluidic, complex permittivity, liquids.

\section{INTRODUCTION}

The microwave spectroscopy is a non-destructive technique which enables to obtain the dielectric properties of a solid, liquid or gaseous material. This technique has been developed of many applicative contexts such as soil moisture measurement or pipeline detection because of its high sensitivity to water, or to verify the quality of materials in agriculture, food and others[1]. More recently, the technique has also made possible to study molecules and biological cells due to their dielectric properties in the microwave range, associated with the use of micrometric and microfluidic technologies [2]-[4]. This scale is indeed compatible with fluids available in small quantities for performing tests and analysis. Nevertheless, applications exist for which the quantity of liquid to be analysed does not constitute a limiting factor with up to millilitres available for analysis. It is consequently interesting to develop new analytical tools, which enables the study of the dielectric properties of such liquids, while relaxing the technological constraints, the cost and finally ubiquitous.

This paper presents a microwave device, which enables to perform the dielectric spectroscopy of millilitres' liquids. This requires the combination of both microwave sensing capability with millifluidic technologies. This makes possible to broaden the sensing capabilities of the microwave spectroscopy and its applications, while using a low cost and less complicated technology for microdevices as well as a transportable Vector Network Analyzer (VNA). The developed technique makes it suitable for various exploitation areas such as health, biology, chemistry or agriculture.

As far as chemistry is concerned, the quantification of molecules in aqueous solution has already been demonstrated at the microscale. Regarding the agriculture of precision, there are strong interests in the development of analytic techniques, which could provide quantitative parameters on the quality of biological entities. Freshness of fishes, fruits or eggs [5]-[9] have already been investigated with various microwave-based sensing techniques, as well as the moisture content in cereal grains or meat [10], [11].

Pertinent examples of chemical and biological samples will consequently be employed for the evaluation of the developed microwave and millifluidic sensor.

This paper first presents the architecture and the fabrication of the microwave and millifluidic sensor. The next section is then dedicated to the modelling of the structure and the extraction of the complex permittivity of the liquid under test. Finally, 2 case studies will be investigated in the fourth section, in order to evaluate the sensitivity of the technique depending on the type of liquids and the associated applicative domain.

\section{ARCHITECTURE AND FABRICATION OF THE MICROWAVE AND MILLIFLUIDIC SENSOR}

The millifluidic and microwave sensor has been designed to extract the complex permittivity of a liquid between 0.1 to 4 GHz. As shown in Fig. 1, it is composed of two parallel coplanar waveguides with identical electrical and dimensional characteristics, except length. The characteristic impedance is $\mathrm{Z}_{0}=50 \Omega$, the widths of the central conductor and the coplanar slot correspond to $\mathrm{w}=0.4 \mathrm{~mm}$ and $\mathrm{s}=0.2 \mathrm{~mm}$ respectively. The coplanar waveguides only differ from their length, which are $\mathrm{L}_{1}$ $=43 \mathrm{~mm}$ and $\mathrm{L}_{2}=56 \mathrm{~mm}$ respectively.

The microwave structure is fabricated on the Rogers RO4003 substrate with a thickness $\mathrm{h}$ of $0.8 \mathrm{~mm}$ and dielectric constant $\varepsilon_{\mathrm{r}}=3.38$ and dissipation factor $\tan \delta=0.0027$. Above these two waveguides are placed two fluidic channels also exhibiting different lengths of 13 and $26 \mathrm{~mm}$ respectively. These channels are made of PDMS, a traditional material for fluidic experiments. 


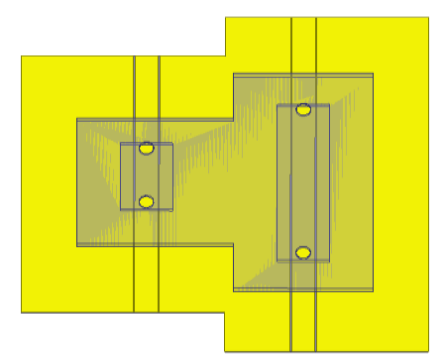

Fig. 1. Schematic of the millifluidic and microwave sensor, which includes 2 coplanar waveguides of different lengths with 2 PDMS fluidic channels on top.

These channels enables to precisely localize the fluids to analyze on the coplanar lines (see Fig. 1). A block of PEEK material is then placed on the PDMS layer and allows the liquid injection within the fluidics channels. The system is screwed to an acrylic support placed under the device in order to maintain by pressure the adhesion of the fluidic channels on the coplanar lines (see Figure 2). The required volume of liquids corresponds to $1.3 \mathrm{ml}$.

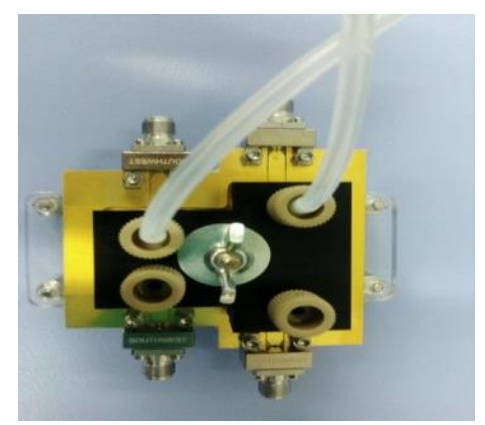

Fig. 2. Photograph of the millifluidic and microwave device, seen from above, with coplanar waveguides, SMA connectors, PEEK block and inlet and outlet tubes.

Next section is related to the modelling of this device to extract the complex permittivity of the liquid under test.

\section{MODELLING}

The modelling method is based on the one developed by Bianco and Parodi [12], and next applied by ReynososHernandez et al. [13] using two micromachined lines transmission with millimetric lengths. This L-L method (for Line-Line) allows extracting the propagation constant from the transition matrix $(\mathrm{T})$ of two nonreflecting lines exhibiting the same characteristic impedance, but different lengths, as shown in Figure 3.

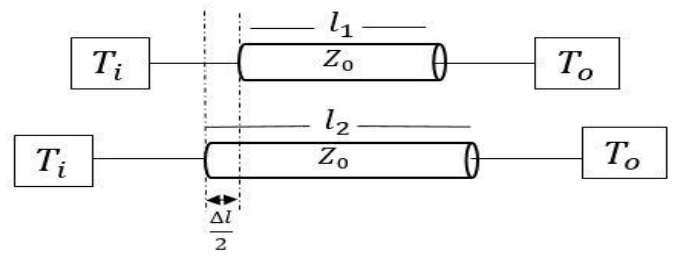

Fig. 3. Schematic of the two transmission lines with the input and output access transmission matrix for the L-L method.

The two-scattering matrix of the input and ouput ports $T_{i}$ and $\mathrm{T}_{\mathrm{o}}$ correspond to the connections between the lines and the cables connected to the VNA. Based on the schematic of Fig. 3, the matrix of the complete structure can be written as:

And

$$
T_{1}=T_{i} T_{L_{1}} T_{o}
$$

$$
T_{2}=T_{i} T_{L_{2}} T_{o}
$$

Where $T_{L_{1}}$ and $T_{L_{2}}$ is the transition matrix of the two lines. After algebraic manipulation of equations (1) and (2), the products $T_{1} T_{2}^{-1}$ and $T_{2} T_{1}^{-1}$ are given respectively by:

$$
\begin{aligned}
& T_{1} T_{2}^{-1}=T_{o}^{-1} T_{L_{2}}^{-1} T_{L_{1}} T_{o}=T_{i} T_{L_{1}} T_{L_{2}}^{-1} T_{i}^{-1} \\
& T_{2} T_{1}^{-1}=T_{o}^{-1} T_{L_{1}}^{-1} T_{L_{2}} T_{o}=T_{i} T_{L_{2}} T_{L_{1}}^{-1} T_{i}^{-1}
\end{aligned}
$$

The addition of equations (3) and (4) is expressed as

$$
\begin{aligned}
\overbrace{\left[T_{1} T_{2}^{-1}+T_{2} T_{1}^{-1}\right]}^{A} & =T_{o}^{-1} \overbrace{\left[T_{L_{2}}^{-1} T_{L_{1}}+T_{L_{1}}^{-1} T_{L_{2}}\right]}^{B} T_{o} \\
& =T_{i}^{-1} \underbrace{\left[T_{L_{1}} T_{L_{2}}^{-1}+T_{L_{2}} T_{L_{1}}^{-1}\right]}_{C} T_{i}
\end{aligned}
$$

Due to equation (5), the terms A, B and C present similar matrices, with the same trace.

$$
\operatorname{tr}(A)=\operatorname{tr}(B)=\operatorname{tr}(C)
$$

If we consider the adapted case with no transmission loss for both $\mathrm{L}_{1}$ and $\mathrm{L}_{2}$ lines, their transition matrix of the $\mathrm{L}_{\mathrm{i}}$ can write as:

$$
T_{L_{i=1,2}}=\left(\begin{array}{cc}
e^{-\gamma l_{i}} & 0 \\
0 & e^{\gamma l_{i}}
\end{array}\right)
$$

Where $\gamma$ is the propagation constant of the lines and $l_{i}$ their length. After manipulating equations (6) and (7), we can express the propagation constant as a function of transition matrices of the system as shown in equation (8). Note the dependence of $\gamma$ as a function of $\Delta l=l_{2}-l_{1}$.

$$
\gamma=\frac{1}{\Delta l} \cosh ^{-1}\left(\frac{\operatorname{tr}\left(T_{1} T_{2}^{-1}+T_{2} T_{1}^{-1}\right)}{4}\right)
$$

On the other hand, the propagation constant may also be written as a function of the frequency f, the light velocity $c$, and the relative permittivity $\varepsilon_{\mathrm{r}}$ as shown in equation (9)

$$
\gamma=j \beta=j \frac{2 \pi f \sqrt{\varepsilon_{r}}}{C} .
$$

Finally, due to equality of equation (8) and (9), the relative permittivity of the propagation constant may be obtained and is described in equation (10):

$$
\varepsilon_{r}=-\left(\frac{\gamma c}{2 \pi f}\right)^{2}
$$


This method has then been used to obtain the permittivity of different liquids placed on top of the two coplanar waveguides for two applicative case studies for sensitivity evaluation.

\section{EXPERIMENTAL EVALUATION OF THE SENSING CAPABILITIES FOR TwO CASE STUDIES}

\section{A. Molecule in aqueous solution}

The first case study corresponds to the detection of bovine serum albumin (BSA) dissolved in water and measured at different concentrations: 10,50 and $100 \mathrm{mg} / \mathrm{ml}$. Each solution has been measured several times, starting from the low BSA concentration to the highest one.

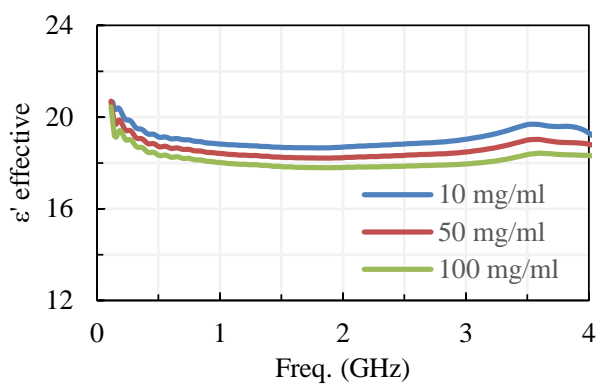

Fig. 4. Real part of the effective permittivity of the structure when loaded with liquids presenting different concentrations of BSA.

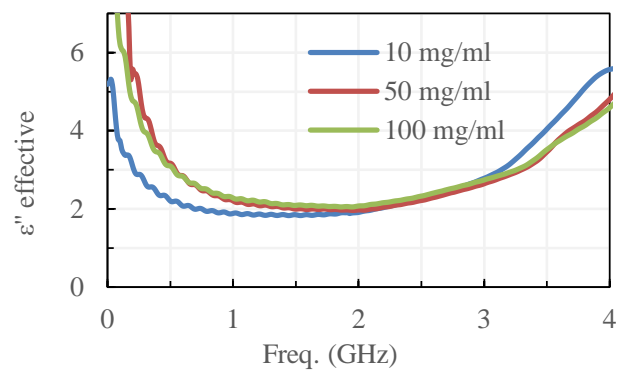

Fig. 5. Imaginary part of the effective permittivity of the structure when loaded with liquids presenting for different concentrations of BSA.

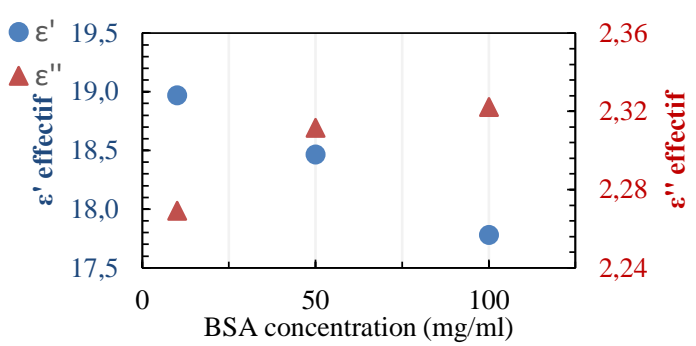

Fig. 6. Real and imaginary parts of the effective permittivity of the structure versus BSA concentration at $3 \mathrm{GHz}$.

The measurements enable a clear discrimination of the liquids with different concentrations of BSA.

The method described in the precedent section has been applied to the measurements. The obtained results, shown in Fig. 4 and Fig. 5, correspond to the real and imaginary parts respectively of the effective permittivity obtained for the different concentrations of BSA as a function of frequency.
A quasi linear relationship between the effective permittivity parameters may be observed in the Fig. 6, which provides the effective permittivity (real and imaginary parts in blue and red respectively) versus the BSA concentration at a fixed frequency of $3 \mathrm{GHz}$.

The standard deviation of the effective permittivity values (both for real and imaginary parts) is presented in Table 1 for the different concentrations of BSA. The dispersion was calculated based on 5 measurements for each solution.

Table 1. Standard deviation of the real and imaginary parts of effective permittivity for different BSA concentrations at $3 \mathrm{GHz}$.

\begin{tabular}{|c|c|c|}
\hline $\begin{array}{c}\text { BSA } \\
\text { Concentrations }\end{array}$ & $\boldsymbol{\sigma} \boldsymbol{\varepsilon}^{\prime}$ & $\boldsymbol{\sigma} \boldsymbol{\varepsilon}^{\prime}$ \\
\hline $10 \mathrm{mg} / \mathrm{ml}$ & 0.02 & 0.003 \\
\hline $50 \mathrm{mg} / \mathrm{ml}$ & 0.01 & 0.003 \\
\hline $100 \mathrm{mg} / \mathrm{ml}$ & 0.057 & 0.016 \\
\hline
\end{tabular}

One may observe a correlation between the permittivity values and the concentration of the measured liquids. A low standard deviation is obtained for the concentrations of 10 and $50 \mathrm{mg} / \mathrm{ml}$, whereas it slightly increases for the $100 \mathrm{mg} / \mathrm{ml}$ concentration. This increase can be attributed to a pollution of the fluidic channel due to the viscosity of the liquid at high concentrations. This standard deviation is however still considered reasonable for rapid, ubiquitous and low-cost experimentations.

\section{B. Microwave measurements of egg constituents}

The second case study is related to the agriculture domain. The chosen material corresponds the extra embryonic liquid of eggs. A previous study indeed targeted to link the moisture content of the yolk or albumen of stored eggs in order to determine the egg freshness as an indicator of quality [5].

Before performing any quality assessment on eggs through microwave spectroscopy, it is mandatory to evaluate the sensitivity of the technique with the targeted samples. Inversely to BSA molecules in aqueous solution, yolk and albumen materials are extracted from various eggs, which are naturally presenting intrinsic dispersions. To do so, different liquids of fertilized duck eggs at 12-day of embryonic development have therefore been measured. The liquid corresponds to the mixing of the extra-embryonic constituents of eggs (removal of the duck embryo and shell egg), while including the membranes, the allantoic, amniotic, and vitellin fluids, and the albumin.

In order to evaluate the repeatability of our measurements of extra-embryonic fluids, 10 samples of different eggs with the same origins and incubation conditions have been analyzed. Due to the high viscosity and strong attachment of egg yolk and albumen to any other materials, a specific cleaning protocol has been developed and applied between each measurement. The extracted effective permittivity was referenced to water as described in equation (11).

$$
\Delta \varepsilon_{e f f}=\varepsilon_{e f f_{\text {Liquid }}} \varepsilon_{\text {eff }} f_{\text {water }}
$$


The real and imaginary parts of the effective permittivity contrast $(\Delta \varepsilon)$ of 10 extra-embryonic fluids samples are shown in Fig. 7 and Fig. 8 respectively.

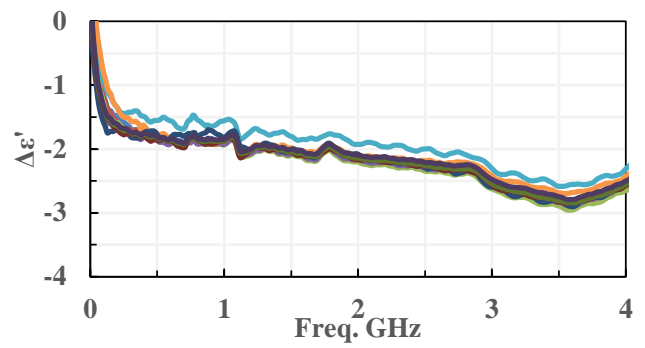

Fig. 7. Contrast of the real part of the effective permittivity of 10 extraembryonic samples fluids referenced to water at $2 \mathrm{GHz}$.

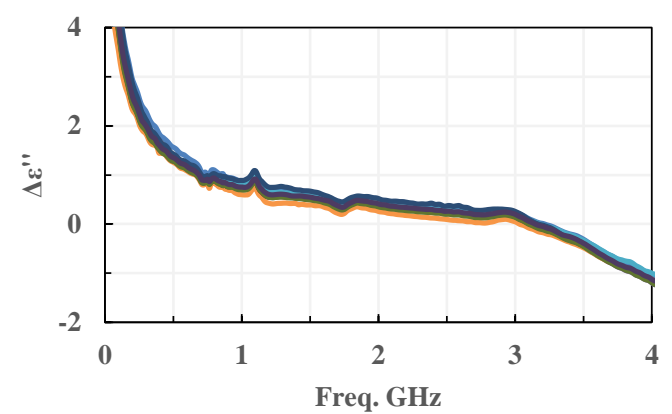

Fig. 8. Contrast of the imaginary part of the effective permittivity of 10 extraembryonic samples fluids referenced to water at $2 \mathrm{GHz}$.

Curves are quite close to each other. For further visibility, the Cole-Cole plot is given in Fig. 9 at $2 \mathrm{GHz}$.

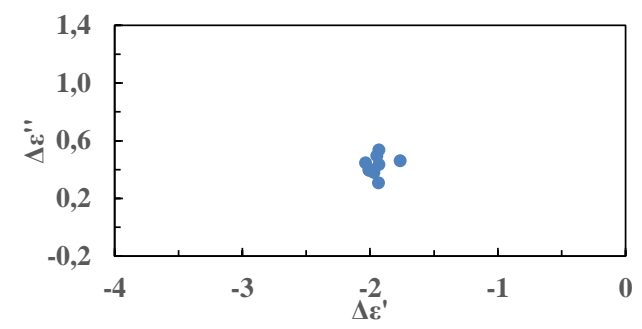

Fig. 9. Cole-Cole plot for 10 extra-embryonic fluids referenced to water at 2 $\mathrm{GHz}$.

The standard deviation $\sigma$ of extra-embryonic fluids measured at $2 \mathrm{GHz}$ is 0.05 for both real and imaginary parts. The differences of $\Delta \varepsilon$ values is partially because of the different biochemical characteristics of different eggs, however the standard deviation remains low. These results allow to validate the fluidic device for the evaluation of extra-embryonic fluids, and constitute the first step toward the analysis of the quality of the eggs fluids.

\section{CONCLUSIONS}

The microwave and millifluidic sensor is presented. A modeling technique based on the L-L method permits to extract the dielectric parameters of the structure loaded with different liquids on the frequency range of 0.1 to $4 \mathrm{GHz}$.

Its sensitivity has been first evaluated with BSA molecules in aqueous solutions with different concentrations. A weak standard deviation of 0.01 on the real part of the permittivity for low concentration has been reached, which validates the developed device and modeling.

These results have been completed with the measurements of extra-embryonic fluids of 10 fertilized duck eggs at 12-day of embryonic development. Even if such a living material presents an intrinsic variability with high viscosity and attachment to any surface, the millifluidic and microwave sensor only exhibits a standard deviation of 0.05 on the real part of permittivity contrast. This result allows us to validate the device and the methodology with the measurement of the dielectric properties of milliliter liquids using a low cost and affordable technology, compatible with many application areas. It notably opens the door towards further investigations for agricultural quality assessment.

\section{REFERENCES}

[1] K. Kupfer, "Elektromagnetische Aquametrie (Electromagnetic Aquametry)," Tm Tech. Mess. Plattf. Für Methoden Syst. Anwendungen Messtech., vol. 69, no. 1/2002, p. 3, 2002.

[2] K. Grenier et al., "Integrated broadband microwave and microfluidic sensor dedicated to bioengineering," IEEE Trans. Microw. Theory Tech., vol. 57, no. 12, pp. 3246-3253, 2009.

[3] Y. Ning et al., "Broadband electrical detection of individual biological cells," IEEE Trans. Microw. Theory Tech., vol. 62, no. 9, pp. 19051911, 2014.

[4] A. Zedek, D. Dubuc, and K. Grenier, "Microwave permittivity extraction of individual biological cells submitted to different stimuli," presented at the Microwave Symposium (IMS), 2017 IEEE MTT-S International, 2017, pp. 865-868.

[5] S. Trabelsi, S. O. Nelson, D. R. Jones, and W. Guo, "Sensing Egg Quality during Storage by Radiofrequency Complex Permittivity Measurement," in Sensors, 2007 IEEE, 2007, pp. 1005-1008.

[6] S. O. Nelson, W. Guo, S. Trabelsi, and S. J. Kays, "Sensing quality of watermelons through dielectric permittivity," in Antennas and Propagation Society International Symposium, 2007 IEEE, 2007, pp. 285-288.

[7] S. O. Nelson, W. Guo, and S. Trabelsi, "Study of fruit permittivity measurements for quality detection," in Instrumentation and Measurement Technology Conference Proceedings, 2008. IMTC 2008. IEEE, 2008, pp. 1009-1011.

[8] S. Redzwan, M. D. Perez, J. Velander, and R. Augustine, "Study of Maturity Fruit Assessment using Permittivity and Microwave Reflectivity Measurements for Quality Classification," in 2018 IEEE Conference on Antenna Measurements \& Applications (CAMA), 2018, pp. 1-3.

[9] K. Ates, S. Ozen, and H. F. Carlak, "The freshness analysis of an apple and a potato using dielectric properties at the microwave frequency region," in Progress In Electromagnetics Research Symposium-Spring (PIERS), 2017, 2017, pp. 1688-1693.

[10] S. O. Nelson, A. W. Kraszewski, S. Trabelsi, and K. C. Lawrence, "Using cereal grain permittivity for quality sensing by moisture determination," in Instrumentation and Measurement Technology Conference, 1999. IMTC/99. Proceedings of the 16th IEEE, 1999, vol. 1, pp. 237-242.

[11] G. Ji, X. Liu, H. Guo, Y. Wang, and L. Yang, "Study of water-injected pork and pork freshness using microwave techniques," in Antennas Propagation and EM Theory (ISAPE), 2010 9th International Symposium on, 2010, pp. 614-617.

[12] B. Bianco and M. Parodi, "Determination of the propagation constant of uniform microstrip lines," Alta Freq., vol. 45, pp. 107-110, 1976.

[13] J. Reynoso - Hernández, C. F. Estrada - Maldonado, T. Parra, K. Grenier, and J. Graffeuil, "An improved method for the wave propagation constant $\gamma$ estimation in broadband uniform millimeter wave transmission line," Microw. Opt. Technol. Lett., vol. 22, no. 4, pp. 268-271, 1999. 